\title{
Simple Technique to Estimate Far-field Intensity of Index Guiding Photonic Crystal Fibers
}

\author{
Dipankar Kundu \\ Assistant Professor, ECE Department, St. Thomas' College of Engineering and Technology, Kolkata, India
}

\begin{abstract}
A simple approach to estimate far-field intensity of index-guiding photonic crystal fibers (PCFs) with given geometrical parameters and wavelength of light is reported. The approach is based on a previously developed simple formulation of the effective cladding indices of the same type of fibers, which is valid in the entire single mode region of such fibers. This region is defined by a prescribed upper limit of a parameter, called relative air-hole diameter. The validity of the approach has already been tested by comparing the results obtained from it with those obtained by other available methods. Since far-field measurements are helpful in characterizing various optical fibers, the far field intensity distribution of the same PCFs is studied here by application of the same formulation. The approach should find wide attention, for its simplicity and easy use, by system designers and users of index-guiding PCFs.
\end{abstract}

Keywords: Index-Guiding Photonic Crystal Fibers, Effective Cladding Index, Effective Index of Fundamental Guided Mode, Far-Field Intensity

\section{INTRODUCTION}

Photonic Crystal Fibers (PCFs), consisting of a pure silica core with a number of periodically spaced air-holes in the cladding region, have attracted great attentions from the last decade of the previous century [1], due to their various noticeable features. These include Endlessly Single Mode (ESM) operation [2], large or ultrasmall Mode Field Diameters (MFDs) and hence corresponding effective areas [3], ultraflattened dispersions [4, 5], high birefringences [6], large nonlinearities [5], supercontinuum generation [7], and so on. The PCFs provide a better optoelectronic tool in various modern fields, such as imaging, telecommunications, spectroscopy, metrology and other new emerging areas [8]. So, these fibers, in near future, are to become ultimately reliable transmission medium for the propagation of electromagnetic waves in suitable context.

PCFs are normally fabricated from undoped silica only [1]. The waveguiding phenomenon in them is due to the airholes in a periodic or regular microstructure, running along the entire length of the fibers. These holes form the cladding region. When the central hole is missing, it creates a defect, which works as the high index core of the waveguide and the waveguide is said to be a solid-core or index guiding PCF. The core region, where light becomes trapped, can support a guided mode. Since the core index is greater than the effective or average cladding index, the principle of light guiding is analogous to Total Internal Reflection (TIR) in Conventional Step Index Fibers (CSIFs) [2]. But, because of the distribution of the lower index air-holes in the cladding region, this guiding mechanism is referred as Modified Total Internal Reflection (m-TIR) [3]. Also, in short wavelength range, a separate waveguiding mechanism, based on Photonic Band Gap (PBG) concept dominates. In this case, the periodically arranged air-holes in PCFs create PBGs, which can prevent fields to propagate in the cladding region for a fixed wavelength range.

To analyze theoretical descriptions of PCFs and predict their propagation characteristics, various numerical methods, like the Effective Index Method (EIM) [2], the multipole method (MPM) [9], the Finite-Element Method (FEM) [10], the Finite-Difference Method (FDM)[11], etc. have been developed and reported in the available literature. The main objective of all these methods is to calculate the effective cladding index of PCFs, accurately, to study those fibers, because of their complex structures and absence of any rotational symmetry in them [12]. This index, which is conventionally denoted by $n_{F S M}$, is the index corresponding to the fundamental cladding mode of the PCF. This particular mode is better known as the Fundamental Space-Filling Mode (FSM) according to EIM over a wide range of wavelength and relevant geometrical parameters- the air-hole diameter and the hole-pitch. The $n_{F S M}$ is the key factor, on which several important modal properties of PCFs, like numerical aperture, MFD, beam divergence, dispersion, bending loss, splice loss, etc. depend [2], ultimately. Almost all the numerical methods, are time consuming and costly. So, it is a practical as well as a pedagogical need for one, using and working with PCFs, to find any simple and general approach to obtain the effective cladding index $n_{F S M}$ of PCFs with a tolerable accuracy and no need of heavy numerical computations. Using EIM, in which a solid-core PCF is treated like a Conventional Step Index Fiber (CSIF), 


\section{International Advanced Research Journal in Science, Engineering and Technology}

Vol. 6, Issue 5, May 2019

with its core and cladding indices replaced by those of silica and the $n_{F S M}$ of the PCF, respectively, a simple and elegant formulation for the said index of the same type of PCFs is presented in [13]. The formulation is valid in the entire single mode region, defined by relative air-hole diameter or $d / \Lambda$-values less than or equal to 0.45 [14]. Also, it enables one to obtain $n_{F S M}$-s, easily and directly, for wide ranges of values of all the opto-geometrical parameters of PCFs. Furthermore, it is adaptable in terms of those parameters to fit to different physical situations. This is the real merit of the approach, which is not obvious for the previous EIMs [2, 12, 15, 16]. The said formulation has been used, effectively, for easy evaluation of various propagation characteristics of the PCF [13]. Since far-field measurements are also helpful in characterizing various optical fibers, it would have been much better and advantageous, if the far-field intensity distribution of solid-core PCFs could be estimated using the same approach as typical examples of field intensity calculations.

In this paper, it is investigated whether the simple formulation for effective cladding index can be used to compute farfield intensity distribution of the index-guiding PCF with an aim to present a simple and novel scheme. Conversely, if one knows the far field measurements of the same type of PCFs, or the far-field pattern is provided, it is possible to determine various important unknown parameters of those fibers [17], like the effective normalized frequency, radius of the core, air hole diameter, spacing between two consecutive air-holes, effective cladding index ( $\left.n_{F S M}\right)$, etc.

\section{ANALYSIS}

\section{A. $\quad$ Preview and Formulation}

An all-silica PCF with triangular lattice of uniform air-holes of diameter $d$, running along the whole length of the fiber, as in Fig. 1, is taken into consideration. The holes are placed symmetrically around a central defect or an omitted air-hole, which is acting as the fiber core. It is made up of solid silica. The air-hole matrix has lattice-constant or holepitch $\Lambda$. This region is assumed to act as the cladding of the fiber. The structure remains same in the longitudinal direction. Since the core-index $n_{C O}$ is greater than the effective or average cladding index $n_{F S M}$, the fiber can guide light by TIR as in case of a CSIF for longer wavelength values and by PBG mechanism for shorter wavelengths.

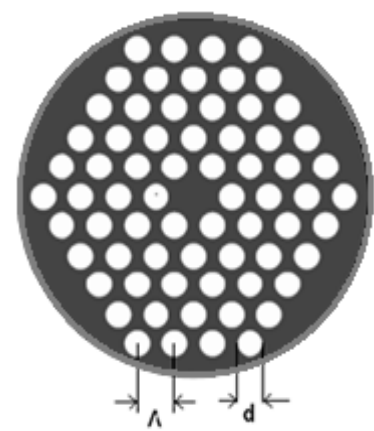

Fig. 1 The index guiding photonic crystal fiber

The propagation constants $\beta$ of the modes, which are guided through the core of the PCF, are given by the relation [2]:

$$
k n_{C O}>\beta>\beta_{F S M}
$$

where $k=2 \pi / \lambda, \lambda$ is the operating wavelength, $\beta_{F S M}$ is the propagation constant of the FSM, the fundamental mode in the infinite photonic crystal cladding without any core or defect. So, $\beta_{F S M}$ is the maximum value of $\beta$ in the cladding region of the concerned PCF.

The effective cladding index or the refractive index of the FSM is given by

$$
n_{F S M}=\frac{\beta_{F S M}}{k}
$$

The procedure to find the index $n_{F S M}$ of the PCF is stated in the Appendix section. 


\section{International Advanced Research Journal in Science, Engineering and Technology}

Vol. 6, Issue 5, May 2019

Now, from the analogy with a CSIF, the effective cladding index $n_{F S M}$ is used to find the effective normalized frequency or $V$-parameter of the PCF, $V_{\text {eff }}$, given as $[2,12,15]$ :

$$
\begin{aligned}
& V_{e f f}=\frac{2 \pi \rho_{e q}}{\lambda}\left(n_{C O}^{2}-n_{F S M}^{2}\right)^{1 / 2} \\
& =\sqrt{U_{e f f}^{2}+W_{e f f}^{2}}
\end{aligned}
$$

where $\lambda$ is the wavelength of the light and $\rho_{e q}$ is the effective core radius, which is considered to be $\Lambda / \sqrt{ } 3$ [12, 15] with

$$
\begin{gathered}
U_{e f f}=\frac{2 \pi \rho_{e q}}{\lambda}\left(n_{C O}^{2}-n_{e f f}^{2}\right)^{1 / 2} \\
\text { and } W_{e f f}=\frac{2 \pi \rho_{e q}}{\lambda}\left(n_{e f f}^{2}-n_{F S M}^{2}\right)^{1 / 2}
\end{gathered}
$$

The $n_{\text {eff }}$ is the effective index of the fundamental guided mode and the two parameters $U_{\text {eff }}$ and $W_{\text {eff }}$ are the effective normalized phase and attenuation constants, respectively, of the chosen PCF, similar to a CSIF.

\section{B. Application to Far-field Pattern}

In the equivalent CSIF of the PCF, the mode field distribution is dependent only on the radial coordinate $r$, indicating that the far field pattern is cylindrically symmetric. The modal solution of the wave propagation through the PCF, which can be used to derive the expression for far field intensity distribution of light wave through it, is obtained from the effective index model, given by [17]

$$
\begin{aligned}
\psi= & \frac{1}{J_{0}\left(U_{\text {eff }}\right)} J_{0}\left(U_{\text {eff }} R\right), R<1 \\
& =\frac{1}{K_{0}\left(W_{\text {eff }}\right)} K_{0}\left(W_{\text {eff }} R\right), \quad R>1
\end{aligned}
$$

where $R=\mathrm{r} / a_{\text {eff }}$ and $a_{\text {eff }}=\Lambda / \sqrt{3}$, as stated earlier.

If the near-field pattern is $\psi(x, y)$ and $u^{\prime}$ denotes the amplitude of far field pattern, it is given as in the following [18]:

$$
u^{\prime}=C \int_{-\infty}^{\infty} \int_{-\infty}^{\infty} \psi(\xi, \eta) e^{i k_{0}(l \xi+m \eta)} d \xi d \eta
$$

where $l=\mathrm{x} / \mathrm{r}, m=\mathrm{y} / \mathrm{r}, l$ and $m$ being the direction cosines of the observation direction, as depicted in Fig. 2 and $k_{0}$ is free space wave number.

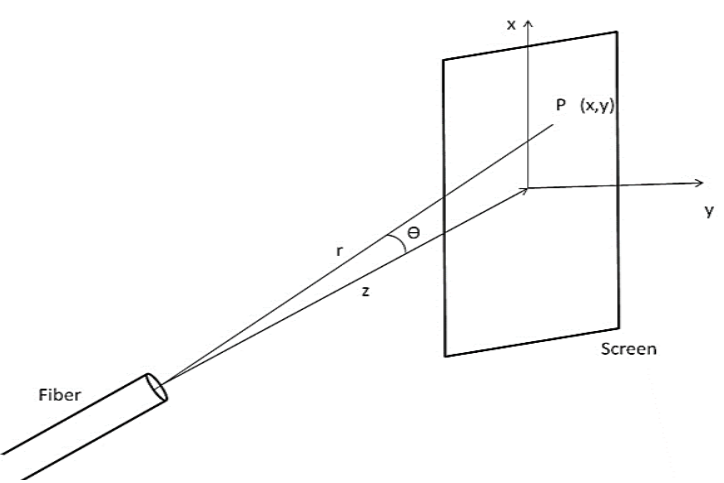

Fig. 2 The point $\mathrm{P}$ is the observation point for far-field pattern at a distance $\mathrm{r}$ from the centre of the exit end of the fiber, where direction of observation makes angle $\theta$ with the z-axis [18] 


\section{International Advanced Research Journal in Science, Engineering and Technology}

Vol. 6, Issue 5, May 2019

The fundamental mode distribution of a circular core optical fiber is cylindrically symmetric. Thus, the far-field pattern is also cylindrically symmetric and the field distribution along the $\mathrm{x}$-axis $(m=0$ and $l=\sin \theta)$ is given by

$$
u^{\prime}=C \int_{-\infty}^{\infty} \int_{-\infty}^{\infty} \psi(\xi, \eta) e^{i k_{0} l \xi} d \xi d \eta
$$

As $\psi$ depends only on coordinate $r$, we can substitute $\xi=r \cos \phi$ and $\eta=r \sin \phi$ and Eq. (8) changes to

$$
\begin{array}{r}
u^{\prime}(\theta)=C \int_{-\infty}^{\infty} \int_{-\infty}^{\infty} r d r \psi(r) e^{i k_{0} r \sin \theta \cos \phi} d \phi \\
=2 \pi C \int_{-\infty}^{\infty} \int_{-\infty}^{\infty} r d r \psi(r) J_{0}\left(k_{0} r \sin \theta\right) d \phi \quad\left[\text { since } J_{0}(\Omega)=\frac{1}{2 \pi} \int_{0}^{2 \pi} e^{i \Omega \cos \phi} d \varphi\right]
\end{array}
$$

If $k_{0} \sin \theta$ in Eq. (9) is replaced by $q$, it can be written as

$$
u^{\prime}(q)=2 \pi C \int_{0}^{\infty} \Psi(r) J_{0}(q r) r d r
$$

Substituting Eq. (6) into Eq. (10), one can obtain

$$
\begin{aligned}
& u^{\prime}(\theta)=\frac{2 \pi C}{J_{0}\left(U_{\text {eff }}\right)}\left[\int_{0}^{a} J_{0}\left(\frac{U_{\text {eff }} r}{a_{\text {eff }}}\right) J_{0}(q r) r d r+\frac{J_{0}\left(U_{\text {eff }}\right)}{K_{0}\left(W_{\text {eff }}\right)} \int_{a}^{\infty} K_{0}\left(\frac{W_{\text {eff }} r}{a_{\text {eff }}}\right) J_{0}(q r) r d r\right] \\
& =\frac{2 \pi C a_{\text {eff }}^{2}}{J_{0}\left(U_{\text {eff }}\right)}\left[\int_{0}^{1} J_{0}\left(U_{e f f} \zeta\right) J_{0}(\alpha \zeta) \zeta d \zeta+\frac{J_{0}\left(U_{\text {eff }}\right)}{K_{0}\left(W_{\text {eff }}\right)} \int_{1}^{\infty} K_{0}\left(W_{\text {eff }} \zeta\right) J_{0}(\alpha \zeta) \zeta d \zeta\right]
\end{aligned}
$$

where $\alpha=q a_{\text {eff }}=a_{\text {eff }} k_{0} \sin \theta$ and $\zeta=\frac{r}{a_{e f f}}$.

For $\theta=0^{0}$, the far field amplitude is given by

$$
u^{\prime}(0)=\frac{2 \pi C a_{\text {eff }}^{2}}{J_{0}\left(U_{e f f}\right)}\left[\int_{0}^{1} J_{0}\left(U_{e f f} \zeta\right) \zeta d \zeta+\frac{J_{0}\left(U_{e f f}\right)}{K_{0}\left(W_{e f f}\right)} \int_{1}^{\infty} K_{0}\left(W_{e f f} \zeta\right) \zeta d \zeta\right]
$$

Using the properties of Bessel function, Eq. (12) reduces to

$$
\begin{aligned}
& u^{\prime}(0)=\frac{2 \pi C a_{e f f}^{2}}{J_{0}\left(U_{e f f}\right)}\left[\frac{J_{l}\left(U_{e f f}\right)}{U_{e f f}}+\frac{J_{0}\left(U_{e f f}\right)}{K_{0}\left(W_{e f f}\right)} \frac{K_{l}\left(W_{e f f}\right)}{W_{e f f}}\right] \\
& =\frac{2 \pi C a_{e f f}^{2}}{J_{0}\left(U_{e f f}\right)} \frac{J_{l}\left(U_{e f f}\right)}{U_{e f f} W_{e f f}^{2}} V_{e f f}^{2} \quad \text { [from the equation for fundamental guided mode in the }
\end{aligned}
$$

equivalent CSIF and Eq. (3b) ]

Thus, the relative far-field pattern is given by

$$
u_{1}(\theta)=\frac{u^{\prime}(\theta)}{u^{\prime}(0)}=\frac{\int_{0}^{l} J_{0}\left(U_{e f f} \zeta\right) J_{0}(\alpha \zeta) \zeta d \zeta+\frac{J_{0}\left(U_{\text {eff }}\right)}{K_{0}\left(W_{\text {eff }}\right)} \int_{0}^{l} K_{0}\left(W_{\text {eff }} \zeta\right) J_{0}(\alpha \zeta) \zeta d \zeta}{\frac{J_{l}\left(U_{\text {eff }}\right)}{U_{\text {eff }} W_{\text {eff }}^{2}} V_{\text {eff }}^{2}}
$$




\section{International Advanced Research Journal in Science, Engineering and Technology}

Vol. 6, Issue 5, May 2019

From the standard integrals, the first integral in the denominator of the right hand side of Eq. (13) is equal to

$$
\frac{U_{e f f} J_{l}\left(U_{e f f}\right) J_{0}(\alpha)-\alpha J_{0}\left(U_{e f f}\right) J_{l}(\alpha)}{U_{e f f}^{2}-\alpha^{2}} \text { for } U_{e f f} \neq \alpha \text { and } \frac{J_{0}^{2}\left(U_{e f f}\right)+J_{l}^{2}\left(U_{e f f}\right)}{2} \text { for } U_{\text {eff }}=\alpha \text {. }
$$

Similarly, the second integral in the same side of that equation is equal to

$$
\frac{W_{e f f} K_{l}\left(W_{e f f}\right) J_{0}(\alpha)-\alpha K_{o}\left(W_{e f f}\right) J_{l}(\alpha)}{W_{e f f}^{2}+\alpha^{2}} .
$$

Substituting these into Eq. (23), the equation reduces to

$$
\begin{gathered}
u_{1}(\theta)=\frac{U_{e f f}^{2} W_{e f f}^{2}}{\left(U_{e f f}^{2}-\alpha^{2}\right)\left(W_{e f f}^{2}+\alpha^{2}\right)}\left[J_{0}(\alpha)-\alpha J_{l}(\alpha) \frac{J_{0}\left(U_{e f f}\right)}{U_{e f f} J_{l}\left(U_{e f f}\right)}\right], \text { for } U_{e f f} \neq \alpha \\
=\frac{U_{e f f}^{2} W_{e f f}^{2}}{2 V_{e f f}^{2} U_{e f f} J_{l}\left(U_{e f f}\right)}\left[J_{0}^{2}(\alpha)+J_{l}^{2}(\alpha)\right], \text { for } U_{e f f}=\alpha
\end{gathered}
$$

Thus, the normalized far field intensity distribution by

$$
\begin{aligned}
I(\theta) & =\left|u_{1}(\theta)\right|^{2}=\left\{\frac{U_{e f f}^{2} W_{e f f}^{2}}{\left(U_{e f f}^{2}-\alpha^{2}\right)\left(W_{e f f}^{2}+\alpha^{2}\right)}\left[J_{0}(\alpha)-\alpha J_{l}(\alpha) \frac{J_{0}\left(U_{e f f}\right)}{U_{e f f} J_{l}\left(U_{e f f}\right)}\right]\right\}^{2}, \text { for } U_{e f f} \neq \alpha \\
& =\left\{\frac{U_{e f f}^{2} W_{e f f}^{2}}{2 V_{e f f}^{2} U_{e f f} J_{l}\left(U_{e f f}\right)}\left[J_{0}^{2}(\alpha)+J_{l}^{2}(\alpha)\right]\right\}^{2}, \text { for } U_{e f f}=\alpha
\end{aligned}
$$

\section{RESULTS AND DISCUSSIONS}

A. Opto-Geometrical Parameters and Procedure

In order to obtain the $n_{F S M}$-values of index guiding PCFs and hence the coefficients in the formulation for the same index, wide ranges of hole-pitch $\Lambda$ and the normalized hole-diameter or relative air-hole size, $d / \Lambda$-values with variations in the wavelength of light $\lambda$, within the range of 0.2 to $2.0 \mu \mathrm{m}$ are considered. The PCF has $n_{C O}=1.45$ and the approach is briefly elucidated in Appendix. Since CSIFs show minimum attenuation loss at $1.55 \mu \mathrm{m}$ and minimum total dispersion at around $1.3 \mu \mathrm{m}$, they are commonly used in this region of wavelengths and the wavelength range, taken here, is including both the minimum loss and minimum dispersion wavelengths. The region can be extended to longer wavelengths, easily, if required. Then with the $n_{F S M}$-values, obtained from the coefficients for different $d / \Lambda$ and particular $\lambda$, the corresponding effective normalized frequencies, $V_{\text {eff }}$ of the same PCF are found, by using Eq. (3a), with core radius $\rho_{e q}=\Lambda / \sqrt{3}$, as mentioned previously. From the obtained $V_{\text {eff }}$-values, the corresponding $U-$ values, or $U_{\text {eff }}$ are computed, as roots, by solving the relevant transcendental equation in a CSIF [18]. Then, the corresponding $n_{\text {eff }}$ and $W_{\text {eff }}$ are obtained from Eqs. (4) and (3b) or (5), accordingly, and the $U_{\text {eff }}, W_{\text {eff }}$ and $V_{\text {eff }}$ are substituted into Eq. (15).

B. Estimation of far field intensity

The normalized far field intensity $I(\theta)$-values are found for a wide range of values of normalized radiation angle $\alpha$ $\left(=a_{\text {eff }} k_{0} \sin \theta\right)$, from 0 to 10 , by using Eq. (15). This range of $\alpha$-values is in consistence with [17]. Fig. 3 shows the plot of far field intensity of the PCF in Decibel (dB) unit as a function of $(\alpha)$ for $d / \Lambda$ values ranging from 0.2 to 0.4 


\section{International Advanced Research Journal in Science, Engineering and Technology}

Vol. 6, Issue 5, May 2019

in steps of 0.1 at two different wavelengths. In fig. $3(\mathrm{a}), I(\theta)$ in $\mathrm{dB}$ is plotted for $\lambda=1.55 \mu \mathrm{m}$ and an arbitrary $\Lambda=3 \mu \mathrm{m}$, whereas fig. 2(b) shows the variation in $I(\theta)$ for $\lambda=1.3 \mu \mathrm{m}$ and same $\Lambda$. Both $\lambda$-values are consistent with [17]. In Fig. 3, shifts are observed in the peaks of the first lobe of the far field intensity patterns. These result from the changes in $V_{\text {eff }}$ due to changes in the index $n_{F S M}$ with change in wavelength $\lambda$ and relative air-hole size $d / \Lambda$. This indicates that the far field intensity of the PCF strongly depends on its $d / \Lambda$ value.

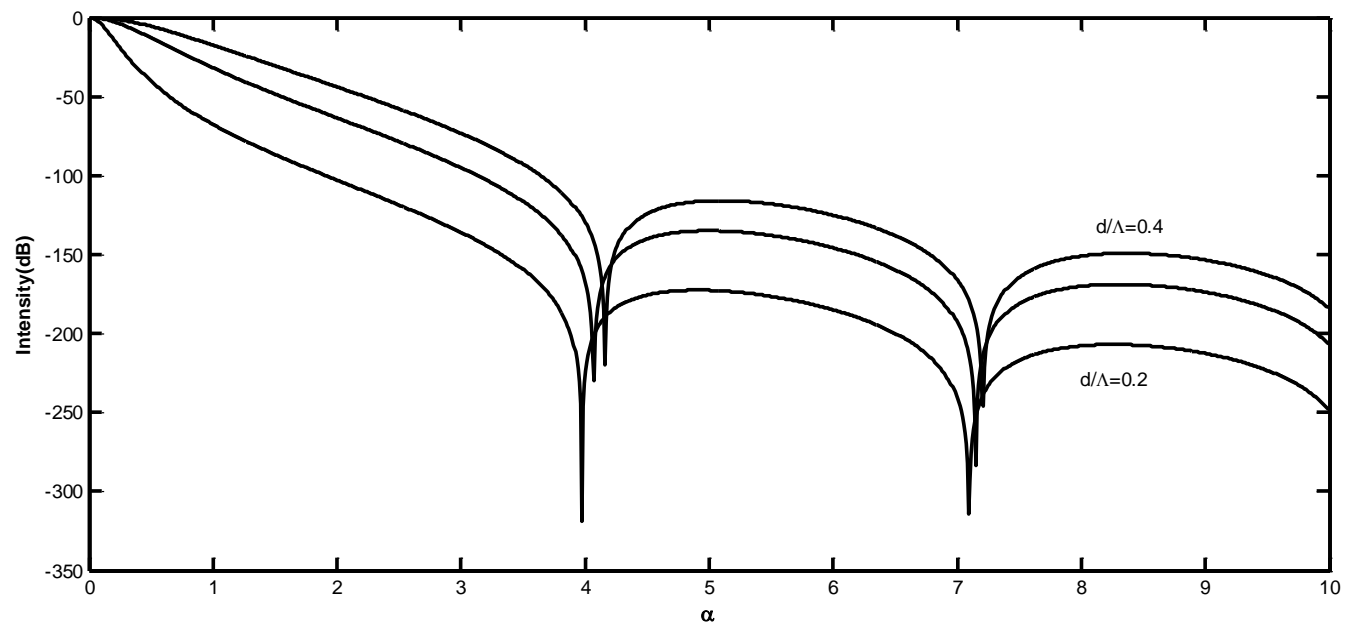

(a)

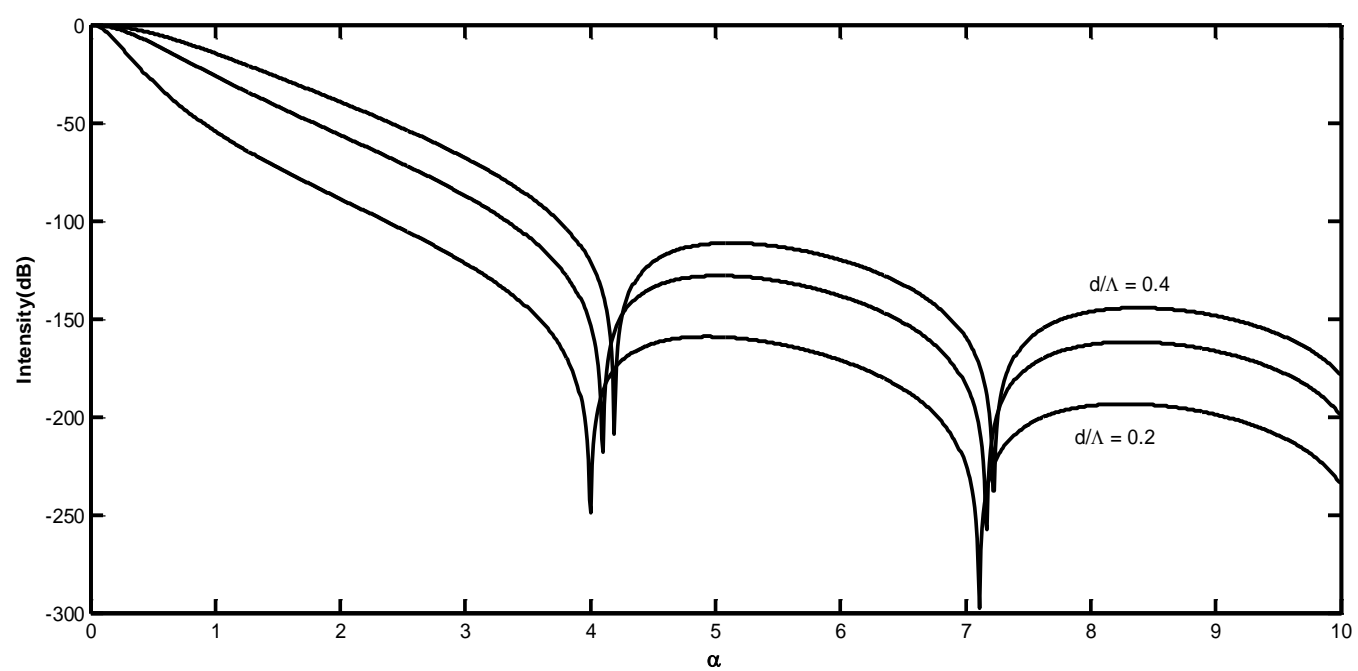

(b)

Fig. 3 Far-field intensity patterns of photonic crystal fibers with $d / \Lambda=0.2,0.3,0.4$ and core radius $\Lambda / \sqrt{3}$ at wavelengths of a) $1.55 \mu \mathrm{m}$ and b) $1.3 \mu \mathrm{m}$

From the physical point of view, if $d / \Lambda$ of an index guiding PCF increases due to increase in $d$-value with $\lambda$ unaltered, the effective cladding index decreases. Since the core-cladding refractive index difference increases, light becomes more confined into the core and the near-field and hence the far-field intensity becomes higher for a specific $\alpha$. The reverse is also true if $d / \Lambda$ is lowered. These are easily observed from both Figs. 3(a) and 3(b). Again, if $\lambda$ decreases with both $d / \Lambda$ and $\alpha$ remaining unchanged, more light remains within the core, resulting in an increase in the far-field intensity. The reverse is also true for an increase in $\lambda$. These are also clearly evident from the above two figures. Thus, the far-field intensity patterns, obtained here, are physically correct and the simple formulation for effective cladding index of solid-core PCFs can be easily used in predicting far-field patterns of the same. 


\section{International Advanced Research Journal in Science, Engineering and Technology}

Vol. 6, Issue 5, May 2019

\section{CONCLUSION}

A simpler and easier analysis to estimate the far field intensity of index guiding PCFs in terms of their opto-geometrical and waveguide parameters is presented. This is based on an appropriate formulation for effective cladding index, which can be oriented in a versatile manner to fit to the desired physical situation. The approach should find wide and ready use by system analysts of PCFs and people, preferring simple approaches with less numerical computations in predicting propagation characteristics of solid-core PCFs.

\section{Appendix: Previous Formulation}

The normalized parameters $v$ and $u$ for the infinite cladding region of the concerned PCF are as given in the following $[1,13]$ :

$$
\begin{gathered}
v=k \Lambda\left(n_{C O}^{2}-1\right)^{1 / 2} \\
\text { and } u=k \Lambda\left(n_{C O}^{2}-\frac{\beta^{2}}{k^{2}}\right)^{1 / 2} \\
\text { with } u^{2}+w^{2}=v^{2} .
\end{gathered}
$$

To find the effective cladding index $n_{F S M}$, a hexagonal unit cell with a basic air-hole at its centre is approximated to a circle in a regular photonic crystal structure [2, 19]. Then from relevant boundary conditions for the fields and their derivatives in terms of appropriate special functions, corresponding to a fixed $v$-value, found from Eq. (A1) for fixed $\lambda$ and $\Lambda$-values, the concerned $u$-value is obtained from the following equation [1]:

$$
\begin{aligned}
& w I_{1}\left(a_{n} w\right)\left[J_{1}(b u) Y_{0}\left(a_{n} u\right)-J_{0}\left(a_{n} u\right) Y_{1}(b u)\right] \\
& +u I_{0}\left(a_{n} w\right)\left[J_{1}(b u) Y_{1}\left(a_{n} u\right)-J_{1}\left(a_{n} u\right) Y_{1}(b u)\right]=0
\end{aligned}
$$

where $a_{n}=\frac{d}{2 \Lambda}, \quad b=\left(\frac{\sqrt{3}}{2 \pi}\right)^{1 / 2}$.

Using Eq. (A4), Russell provided a polynomial fit to $u$, valid only for $d / \Lambda=0.4$ and $n_{C O}=1.444$ [1]. That is required to be simpler and applicable for all $d / \Lambda$ values of practical interest in the entire single mode region of a PCF, for which, $d / \Lambda \leq 0.45$. Then the roots of Eq. (A4) or the $u$-values are obtained for different $d / \Lambda$ and $\lambda$-values, taking $n_{C O}=1.45$, as in $[12,15]$ and the corresponding $n_{F S M}$-s are obtained by replacing $\beta / k$ in Eq. (A2) with $n_{F S M}$. Then the following equation is written [13]:

$$
n_{F S M}=A+B \lambda+C \lambda^{2}
$$

where $A, B$ and $C$ are three different optimization parameters, dependent on both the relative hole-diameter $d / \Lambda$ and the hole-pitch $\Lambda$. The $A, B$ and $C$-values are found by least square fitting of $n_{F S M}$ in terms of $\lambda$ to Eq. (A5) for a fixed $d / \Lambda$ with variations in $\lambda$. Then, different $A, B$ and $C$-values are simulated for various $d / \Lambda$ in the endlessly single mode region of the PCF, which result in the empirical relations for $A, B$ and $C$ in Eq. (A5), in terms of $d / \Lambda$, as given below [13]:

$$
\begin{gathered}
A=A_{0}+A_{1}\left(\frac{d}{\Lambda}\right)+A_{2}\left(\frac{d}{\Lambda}\right)^{2} \\
B=B_{0}+B_{1}\left(\frac{d}{\Lambda}\right)+B_{2}\left(\frac{d}{\Lambda}\right)^{2} \\
C=C_{0}+C_{1}\left(\frac{d}{\Lambda}\right)+C_{2}\left(\frac{d}{\Lambda}\right)^{2}
\end{gathered}
$$




\section{International Advanced Research Journal in Science, Engineering and Technology}

Vol. 6, Issue 5, May 2019

where $A_{i}, B_{i}$ and $C_{i}(i=0,1$ and 2) are the optimization parameters for $A, B$ and $C$ respectively, which are same as those in Eq. (A5).

Computing these $A, B$ and $C$ from Eq. (A6-A8), $n_{F S M}$-s can be directly obtained for any $d / \Lambda$ and $\lambda$-values in the endlessly single mode region of PCFs using Eq. (A5). Then various $A_{i}, B_{i}$ and $C_{i}(i=0,1$ and 2) are simulated with different $\Lambda$-values and the following empirical relations are written in terms of $\Lambda$ [13]:

$$
\begin{array}{r}
A_{i}=A_{i 0}+A_{i 1} \Lambda+A_{i 2} \Lambda^{2} \\
B_{i}=B_{i 0}+B_{i 1} \Lambda+B_{i 2} \Lambda^{2} \\
\text { and } \quad C_{i}=C_{i 0}+C_{i 1} \Lambda+C_{i 2} \Lambda^{2}
\end{array}
$$

\begin{tabular}{|c|c|c|c|c|c|c|c|c|c|}
\hline & \multicolumn{3}{|c|}{$i=0$} & \multicolumn{3}{|c|}{$i=1$} & \multicolumn{3}{|c|}{$i=2$} \\
\hline & $\mathbf{j}=\mathbf{0}$ & $j=1$ & $\mathrm{j}=\mathbf{2}$ & $\mathbf{j}=\mathbf{0}$ & $\mathrm{j}=1$ & $\mathrm{j}=2$ & $\mathbf{j}=\mathbf{0}$ & $\mathrm{j}=1$ & $\mathrm{j}=2$ \\
\hline $\mathbf{A}_{\mathrm{ij}}$ & 1.448716 & 0.000626 & $\overline{-} .000055$ & 0.019655 & $\overline{-} \mathbf{0} 006738$ & 0.000518 & 0.016806 & $\overline{-}-003600$ & 0.000210 \\
\hline $\mathbf{B}_{\mathrm{ij}}$ & $\begin{array}{l}- \\
0.001844\end{array}$ & $\begin{array}{l}- \\
0.000385\end{array}$ & 0.000066 & $\begin{array}{l}- \\
0.058269\end{array}$ & 0.021157 & $\overline{-} .001671$ & $\overline{0} .122305$ & 0.030405 & $\begin{array}{l}- \\
0.001969\end{array}$ \\
\hline$C_{i j}$ & 0.001908 & $\overline{-} .000295$ & 0.000006 & 0.016393 & $\overline{-} \mathbf{0} .007367$ & 0.000636 & $\overline{0}-021490$ & 0.004703 & $\overline{0} .000319$ \\
\hline
\end{tabular}

where for each $i$, in the values of $A_{i j}, B_{i j}$ and $C_{i j}, j$ has values 0,1 and 2 .

Table I: Font Sizes for Papers Values of Coefficients for $n_{F S M}$ with $n_{C O}=1.45$

\section{REFERENCES}

[1]. P. St. J. Russell, "Photonic-crystal fibers," J. Lightwave Tech., vol. 24, pp. 4729-4749, Dec. 2006.

[2]. T. A. Birks, J.C. Knight, \& P.St.J.Russell, "Endlessly single-mode photonic crystal fiber,” Optics Letters, vol. 22, pp. 961-963, Jul. 1997.

[3]. J. C. Knight, T. A. Birks, R. F. Cregan, P. St. J. Russell, and J. -P. de. Sandro, "Large mode area photonic crystal fibre," Electronics Letters, vol. 34, pp. 1347-1348, Jun. 1998.

[4]. N. G. R. Broderick, T. M. Monro, P. J. Bennett, and D. J. Richardson, "Nonlinearity in holey optical fibers: measurement and future opportunities," Optics Letters, vol. 24, pp. 1395-1397, Oct. 1999.

[5]. A. Ferrando, E. Silvestre, J. J. Miret, and P. Andre's, "Nearly zero ultraflattened dispersion in photonic crystal fiber," Optics Letters, vol. 25, pp. 790-792, Jun. 2000 .

[6]. A. Ortigosa-Blanch, J. C. Knight, W. J. Wadsworth, J. Arriaga, B. J. Mangan, T. A. Birks, and P. St. J. Russell, "Highly birefringent photonic crystal fibers," Optics Letters, vol. 25, pp. 1325-1327, Sep. 2000.

[7]. J. K. Ranka, R. S. Windeler, and A. J. Stentz, "Visible continuum generation in air-silica microstructure optical fibers with anomalous dispersion at $800 \mathrm{~nm}$," Optics Letters, vol. 25, pp. 25-27, Jan. 2000.

[8]. S. K. Varshney, \& R. K. Sinha, “Spectral Response of Bend Loss in Photonic-Crystal Fibers,” Laser Phy, vol.14(5), pp.756-759, 2004.

[9]. T. P. White, R. C. McPhedran, L. C. Botten, G. H. Smith, and C. M. de. Sterke, "Calculations of air-guided modes in photonic crystal fibers using the multipole method," Opt. Express, vol. 9, pp. 721-732, Dec. 2001.

[10]. F. Brechet, J. Mercou, D. Pagnoux, and P. Roy, "Complete analysis of the characteristics of propagation into photonic crystal fibers by the finite element method," Opt. Fiber Technol., vol. 6, pp. 181-191, Apr. 2000

[11]. Y. J. He, and F. G. Shi, "Finite-difference imaginary-distance beam propagation method for modeling of the fundamental mode of photonic crystal fibers," Opt. Commun., vol. 225, pp. 151- 156, Sep. 2003.

[12]. K.Saitoh, \& M.Koshiba, "Empirical relations for simple design of photonic crystal fibers,” Opt. Express, vol.13, pp.267-274, Jan. 2005.

[13]. D. Kundu, and S. N. Sarkar, "Prediction of propagation characteristics of photonic crystal fibers by a simpler, more complete and versatile formulation of their effective cladding indices," Optical Engineering, vol. 53, pp. 056111-1-056111-6, May 2014.

[14]. J. Ju, W. Jin, Y. L. Ho, and M. S. Demokan, “A simple method for estimating the splice loss of photonic crystal fiber/single-mode fiber," Microwave and Optical Technology Letters, vol. 42, pp. 171-173, May 2004.

[15]. M.Koshiba, \& K.Saitoh, "Applicability of classical optical fiber theories to holey fibers," Optics Letters, vol 29(15), pp.1739-1741, Aug. 2004.

[16]. T. Sorensen, J. Broeng, A. Bjarklev, E. Knudsen, and S. E. B. Libori, "Macro-bending loss properties of photonic crystal fibre," Electronics Letters, vol. 37(5), pp. 287-289, Mar. 2001.

[17]. S. K.Varshney, and R. K. Sinha, "Characterization of photonic crystal fibers from far field measurements," Journal of Microwaves, Optoelectronics and Electromagnetic Applications, vol. 2(6), pp. 32-42, Dec. 2002.

[18]. A. Ghatak, \& K. Thyagarajan, Introduction to Fiber Optics, $1^{\text {st }}$ South Asian ed., Cambridge University Press India Pvt. Ltd.1999, Repri- 2011.

[19]. R. K. Sinha, and A. D. Varshney, "Dispersion properties of photonic crystal fiber: comparison by scalar and fully vectorial effective index methods," Opt. Quantum Electron. vol. 37(8), pp. 711-722, Jun. 2005. 